\title{
Análise geométrica da forma da beleza da mama e da forma de prótese baseado na proporção Phi: aplicação prática
}

\author{
Geometric analysis of the shapes of the beautiful breast and breast implants \\ based on the golden ratio (Phi): practical application
}

Antonio Roberto Bozola ${ }^{1}$
Franco Marcelo Longato ${ }^{2}$
Adriana Parro Bozola ${ }^{3}$

Trabalho realizado no Serviço de Cirurgia Plástica do Hospital de Base da Faculdade de Medicina de São José do Rio Preto, São José do Rio Preto, SP, Brasil.

Artigo submetido pelo SGP (Sistema de Gestão de Publicações) da RBCP.

Artigo recebido: $3 / 1 / 2011$

Artigo aceito: $15 / 2 / 2011$

\begin{abstract}
RESUMO
Introdução: Os autores observaram resultados insatisfatórios e inconstantes na forma final de mamas após inclusão de próteses de formatos convencionais em casos de hipomastias, havendo poucos estudos a respeito de formas de mamas e de próteses. Método: Descrevem a proporção Phi (1/1.618...), historicamente conhecida como proporção áurea ou divina, e seus conceitos. Argumentam sua importância nas formas em geral, registrada no sistema límbico humano como valor de beleza, harmonia e equilíbrio de proporções. Propõem uma anatomia da beleza da forma da mama em visão tridimensional (visão frontal, lateral e vertical), geometrizada e baseada em Phi. Classificam as mamas quanto à forma incluindo as diferentes formas de hipomastias. Propõem nova forma de prótese mamária, contendo a proporção Phi e coberta com poliuretano. Escolhem as próteses a serem utilizadas por meio de uma medida vertical do tórax da paciente em visão frontal, e da classificação das hipomastias. Resultados: Dez pares de próteses foram especialmente confeccionados e implantados em dez pacientes que foram acompanhadas por 12 meses, apresentando bons resultados finais de forma e volume. Conclusão: É possível estabelecer visão geométrica e tridimensional das mamas bonitas quanto à forma, baseada na proporção Phi, e próteses com a forma também confeccionadas sob a mesma proporção para corrigir as hipomastias. Além de maneira simples de escolha das próteses, o método proporciona a obtenção de resultados bons e homogêneos de formas e volumes mamários, reduzindo subjetividade.
\end{abstract}

Descritores: Implante Mamário. Próteses e Implantes. Mama/cirurgia.

\begin{abstract}
Introduction: The authors observed dissatisfactory and inconsistent results in the final shape of the breast after placement of conventional prostheses in cases of hypomastia, and few studies regarding breast or implant shapes. Methods: They described Phi (1/1.618..), historically known as the golden or divine ratio. They argued its importance to shapes in general, registered in the human limbic system as the value of beauty, harmony and balance of proportion. They proposed an anatomy of the beautiful breast shape from a three-dimensional, geometric view (frontal, lateral and vertical views), based on Phi. They classify the breasts according to shape, including the different hypomastias. They propose a new shape for breast implants, which contains the ratio Phi, covered with polyurethane. The specific prosthesis to be used is chosen according to the vertical measure of the patient's thorax from the frontal view and the type of hypomastia. Results: Ten pairs of breast prostheses were specially manufactured and implanted in ten patients who were accompanied for 12 months, presenting good final results in terms of shape and volume. Conclusion: Based on Phi, it is possible to establish a geometric, three-dimensional view of the beautiful breast in terms of shape as well as implants with the same shape, manufactured also using Phi, to correct hypomastias. In addition the method offers a simple way to choose implants, obtaining good and consistent results of breast shape and size, and reducing subjectivity.
\end{abstract}

Keywords: Breast Implantation. Prostheses and Implants. Breast/surgery.

\footnotetext{
1. Professor Doutor em Cirurgia Plástica, Regente do Serviço de Cirurgia Plástica do Hospital de Base, São José do Rio Preto, SP, Brasil

2. Cirurgião Plástico - Itália.

3. Cirurgiã Dentista, São José do Rio Preto, SP, Brasil.
} 


\section{INTRODUÇÃO}

Além da lactação como função primordial, as mamas sempre foram analisadas sob perspectiva sexual e estética ao longo dos séculos. A sua exposição e o seu resguardo pudico também passou e tem passado por fases culturais e geográficas. Tomou aspectos nunca antes conhecidos, a partir do desenvolvimento das próteses de silicone para solucionar os problemas de forma e volume num percentual elevado de mulheres insatisfeitas com as desproporções das suas mamas.

No arsenal da cirurgia plástica, a receptividade destes procedimentos nunca parou de aumentar em função do custo e do benefício que têm proporcionado.

A diversidade de forma e volume das mamas, relacionada a genética, gravidez, variação de peso do corpo e estrutura glândulo-adiposa, tem motivado continuamente as pacientes a buscar na cirurgia a devida correção estética.

A cirurgia das mamas recebeu ao longo das décadas um numeroso acervo de técnicas, não existindo uma única que pudesse ser aplicada a todos os tipos.

Algumas vezes, a forma bonita da mama não existe, o volume é insuficiente e a implantação da prótese mamária é necessária e solicitada. Volume, forma e ptose são diagnósticos diferentes e devem ser corrigidos individualmente em implantação de próteses mamárias. Correntemente, existem resultados insatisfatórios de forma que são referidos pelas pacientes com seus aumentos mamários. Uma delas é a ausência do pólo superior, comuns em pacientes magras. $\mathrm{O}$ colo mamário permanece vazio após a aplicação do implante e o cirurgião plástico não pode executar nada melhor porque as formas de próteses são inadequadas.

Historicamente não há muitas publicações contendo informações suficientes sobre formas de próteses. Foram discutidas apenas por Vázquez ${ }^{1}$. Outros autores referiram perfil de próteses ${ }^{2,3}$ ou seus perfis anatômicos ${ }^{4,5}$. Entender a forma das mamas e a forma das próteses é importante para obter sempre bom resultado final aos olhos de quem admira. O resultado final em mamaplastias de aumento é o volume e a forma da mama mais o volume e a forma da prótese. Na reconstrução das mamas, a forma e o volume final dependem somente da prótese mamária usada.

Quais são as causas de uma mama ter forma feia ou bonita? É conveniente entender que espécie de características a mama bonita contém. O que pode o cirurgião plástico identificar na forma anatômica bonita da mama e reproduzir na implantação de próteses mamárias? Desafortunadamente, os estudos de formas de próteses mamárias foram prioridade apenas das indústrias, com poucas sugestões científicas dos cirurgiões.

Se um padrão de beleza da forma da mama for estabelecido, será possível reproduzi-lo nos implantes mamários de qualquer volume e evitar maus resultados estéticos.
Este trabalho propõe otimizar os resultados de forma em implantação de próteses mamárias estudando:

- a forma das mamas sob visão tridimensional e a perspectiva geométrica com base na proporção Phi, estabelecendo uma anatomia para a forma bonita da mama padrão;

- as diferentes formas de hipomastias;

- uma nova forma de prótese, com base na proporção Phi.

Além dos critérios atualmente em uso entre os especialistas, cremos trazer mais um elemento coadjuvante prático e acadêmico. A introdução de Phi, que é matematicamente reprodutível, na obtenção da qualidade estética dos resultados.

\section{Phi - História e conceitos atuais e sua importância nas formas}

Nossas reações à beleza e à estética se construíram desde o período pré-histórico, frente às pressões adaptativas da seleção natural na espécie humana ${ }^{6}$. Reagimos instintivamente, o que na Psicologia Jungliana é chamado de Arquétipo $^{7}$. Traços geométricos podem ser reconhecíveis por circuitos cerebrais moldados pela atuação da seleção natural no cérebro humano ${ }^{8,9}$, e que atuam como mecanismo prévio de disciplina ${ }^{10}$.

Somente uma proporção matemática é repetidamente referida, desde a antiguidade até os dias atuais, por estar contida em formas geralmente bonitas. A proporção Phi (1/16180339887 ...) (Figura1). Ela sempre existiu no universo da física e da matemática. Quando a proporção Phi foi primeiramente descrita ou aplicada, desconhece-se. Os filósofos e matemáticos gregos Platão, Aristóteles, Pythagoras e Euclides a estudaram ${ }^{11-16}$ e observaram que criações que a respeitassem demonstravam harmonia ${ }^{6}$ e beleza. Os egípcios utilizaram a proporção Phi na construção das pirâmides ${ }^{15}$. O escultor grego Phidias muito a utilizou, inclusive no Parthenon. Assim, no início do século XX, ela foi chamada Phi pelo matemático americano Mark Barr: a primeira letra da primeira parte do nome do escultor e arquiteto grego Phidias ${ }^{10}$.

Phi também foi descrita por Leonardo de Pisa (Fibonacci), que a referiu na sua sequência matemática. Leonardo da Vinci chamou-a de proporção áurea e a usou para a obra do Homem Vitruviano. Kepler chamou-a de divina. Paccioli chamou-a de divina proporção ${ }^{8,9,16}$. Botânicos a encontram na filotaxia ${ }^{17}$. A proporção Phi é observada em partituras de músicas clássicas ${ }^{12}$. Acredita-se que Phi esteja registrada no sistema límbico humano como valor de eficiência, beleza, harmonia e equilíbrio ${ }^{10}$. É de reconhecimento instintivo, herança estética do processo evolutivo da humanidade. Desde Da Vinci, estudos em formas de face ${ }^{7,17,18}$ e também de Psico$\operatorname{logia}{ }^{16,19}$ foram desenvolvidos sobre as preferências humanas por estruturas que contenham esta proporção. 
Foram selecionadas três figuras geométricas baseadas na proporção Phi para servirem de modelos no estudo geométrico e estético das mamas:

1. Ovoide (Figura 2) - A projeção num único plano de um ovoide constituído por dois círculos parcialmente superpostos, qualquer que sejam suas dimensões. Uma vez aplicada a proporção Phi (1/1.618) nos seus eixos verticais, como na Figura $2 \mathrm{~A}$ (AD está para $\mathrm{BD}$ assim como BD está para $\mathrm{CD}$ ), obteremos sempre a mesma forma, a qual poderíamos denominar de "ovoide áureo";

2. Triângulo áureo isóscele - reconhecido assim ao longo da história de Phi (Figura 3). Suas subdivisões sequenciais mantêm a mesma forma e ângulos infinitamente;

3. Quatro triângulos áureos unidos por um de seus lados mais próximos e ápices formam uma "pirâmide áurea" (Figura 4A). Se girados no eixo vertical, formam o que se poderia denominar "cone áureo" (Figura 4B).

O ovoide foi aplicado na visão frontal da base da mama bonita e das próteses, o triângulo na visão lateral, e o cone na visão vertical.

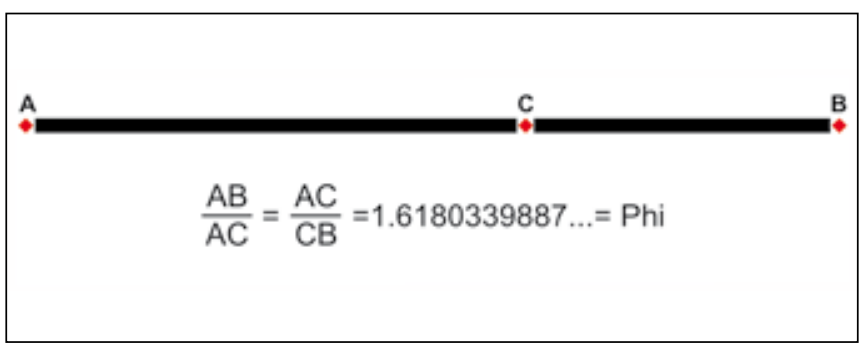

Figura 1 - A proporção Phi (1/1.6180339887...), denominada de áurea ou divina, como fator matemático de beleza em várias áreas do conhecimento e que sensibiliza os sentidos humanos.

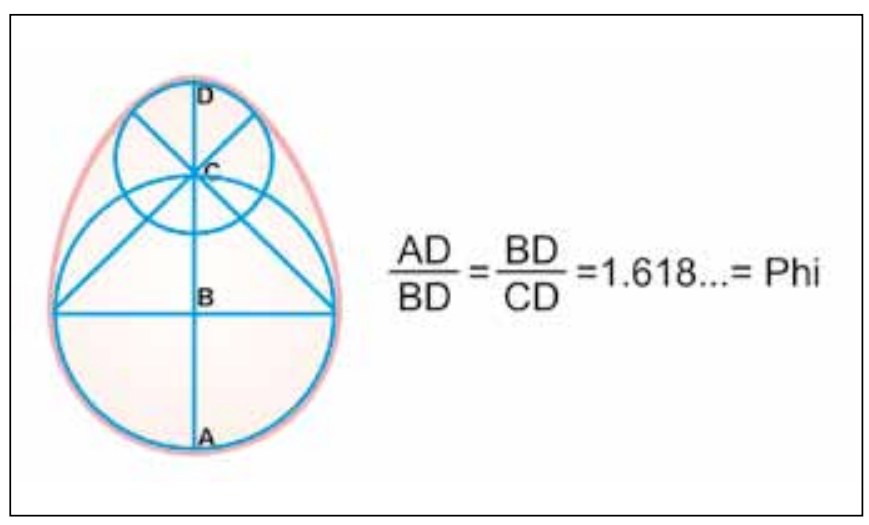

Figura 2 - Ovoide áureo com a proporção Phi proposta pelos autores como base para análise de implantação da mama no tórax.

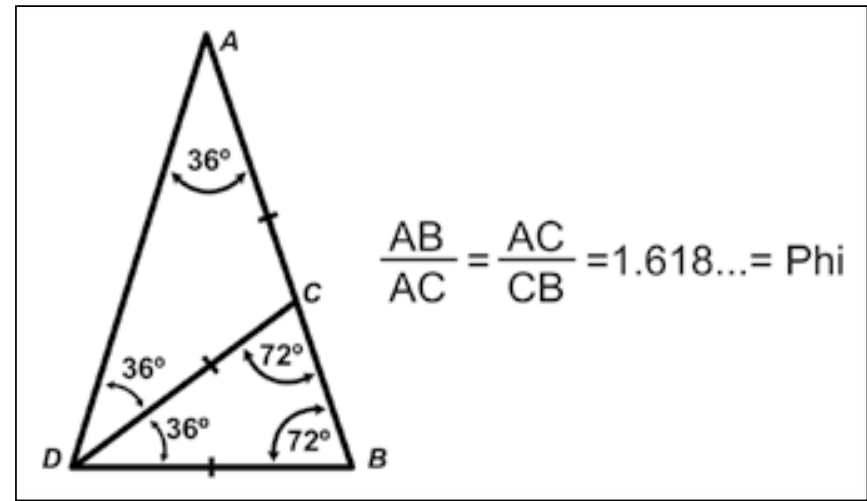

Figura 3 - O reconhecido triângulo áureo com a proporção Phi proposta para análise do perfil da mama bonita.



Figura 4-A: Quatro triângulos áureos com a proporção Phi unidos do ápice à base, obtendo uma pirâmide áurea. B: $A$ pirâmide girada em seu eixo vertical, obtendo um cone áureo, para análise da projeção da mama.

\section{MÉTODO}

Proporções e anatomia da beleza da forma da mama

A forma bonita da mama pode ser analisada sob visão tridimensional (frontal, lateral e vertical), onde em cada uma se refletem também as possíveis distorções estéticas.

- Visão frontal: considerando a forma da mama como um cone irregular, sua implantação tem o diâmetro horizontal menor que o vertical, na proporção de 1 para 1.3. Esta proporção é consequência da aplicação de Phi no eixo vertical (Figura 5A), com a forma do ovoide áureo. $\mathrm{O}$ eixo $\mathrm{ABCD}$ é o meridiano mamário (breast meridian line - BML) e está compreendido entre duas outras linhas horizontais. A linha mamária horizontal inferior (lowest horizontal breast line - LHBL) une os sulcos inframamários na confluência deles com os meridianos mamários (pontos I - Figura 5A). A linha mamária horizontal superior (highest 
horizontal breast line - HHBL) une a parte mais alta das duas pregas axilares anteriores. A medida vertical entre essas duas linhas é individual para cada paciente, determinando a extensão vertical da base da mama. Aplicando a proporção de 1 para 1.3 sobre ela, obteremos a proporcional e consequente extensão lateral. Na visão frontal da mama, quando há flacidez de pele, haverá ptose, o eixo vertical e o eixo horizontal tendem a inverter as proporções, o vertical reduz e o horizontal aumenta, o colo esvazia. Os pontos I não se alteram.

- Visão lateral: o esqueleto torácico normal, na região anterior esternocostal, tem anatomicamente uma inclinação aproximada de 18 graus em relação ao eixo vertical do corpo, na região de implantação das mamas. Quando o triângulo áureo é inclinado sob esse ângulo em relação ao plano vertical, se assemelhará à mama bonita de perfil (Figuras 5B e 5C). Uma mama normal sem ptose, em visão lateral, está acima do plano horizontal A (LHBL) (Figuras 6A e 6B). O plano A coincide com os pontos I (cruzamento do meridiano mamário com o sulco inframamário). Outro plano horizontal (M) cruza o ápice do cone mamário, e divide a mama em dois segmentos: superior e inferior. Quando ele cruza o meridiano da mama, determina o ponto $\mathrm{M}$, onde em geral encontra-se a papila (ela e a aréola podem estar ectópicas, fora do ápice do cone). Do ápice do cone até o ponto I as mamas têm medidas ideais entre 7 e 12 $\mathrm{cm}$. Estas medidas podem variar de acordo com países, épocas e modismos. Medidas menores vão determinar mamas de menores volumes e projeção e vice-versa. Mamas menores tendem para $7 \mathrm{~cm}$ e maiores, para $12 \mathrm{~cm}$. O segmento mamário superior do ápice do cone até a highest horizontal breast line (HHBL) mede 1.618 vezes a medida do segmento inferior. É a proporção Phi novamente (Figuras 6A e 6B).

- Visão vertical: Na visão vertical individual, quando a paciente olha para si mesma de cima para baixo (Figuras 7A e 7B), a mama normal e bonita está entre duas linhas verticais: a vertical lateral breast line (VLBL), e a vertical medial breast line (VMBL). A primeira coincide com a linha axilar anterior; e a segunda está situada 1 a $2 \mathrm{~cm}$ lateralmente a linha médio-esternal (Figuras 7A e 7B). Aplicando a análise geométrica na visão vertical, observa-se que unindo quatro triângulos áureos (Figura 4A) pelos seus lados mais próximos e pelos ápices forma-se uma pirâmide áurea. Se girarmos essa pirâmide sobre seu eixo vertical, resultará em um cone áureo (Figura 4B). Este apresenta a relação de 1 para 1.618 entre sua altura e base e assemelha-se a uma mama bonita vista pela visão vertical. Se as linhas da base ao ápice forem curvadas se parecerá com a mama bonita da visão vertical (Figura 8).

Os autores consideram que a proporção Phi não deve ser fixada como medida exata. Mas como valor referencial e incontestável para se estabelecer um padrão anatômico de forma de mama bonita, que seja matematicamente reprodutível, pois uma mama também é bonita sob a proporção de 1 para 1.5 .

Uma mama pode ter forma bonita e pequeno volume quando nos extremos dos diâmetros horizontal e vertical não toca as linhas horizontais e verticais descritas e estabelecidas no tórax como seus limites.

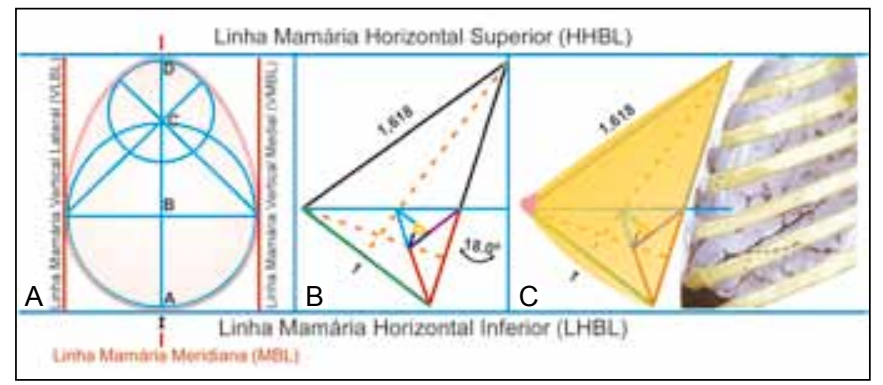

Figura 5-A: O ovoide áureo como a base da mama bonita na visão frontal, contido entre as quatro linhas mamárias que delimitam a extensão da base e a posição da mama bonita no tórax. B: A inclinação do triângulo áureo em 18 graus igual à inclinação do tórax na área de implantação da mama. $\boldsymbol{C}:$ A mama estilizada como o triângulo áureo e inclinada 18 graus ajustada ao tórax.

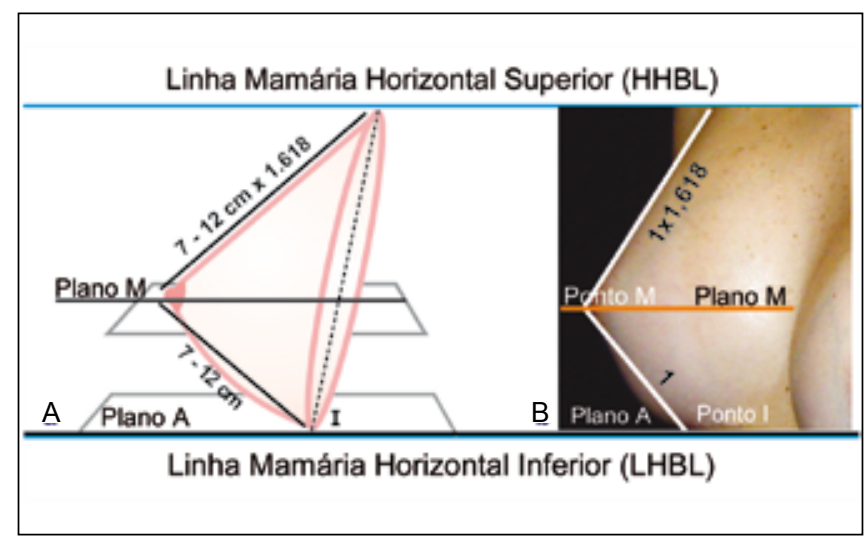

Figura 6 - A: Esquema em visão lateral da mama de forma anatômica bonita, com a proporção Phi entre as medidas do pólo inferior e superior e, consequentemente, as outras. O plano $A$ passando pelo ponto I e o plano M passando pelo ápice do cone. B: A mama anatomicamente bonita com as mesmas características e proporções geométricas do esquema da Figura $6 \mathrm{~A}$. 


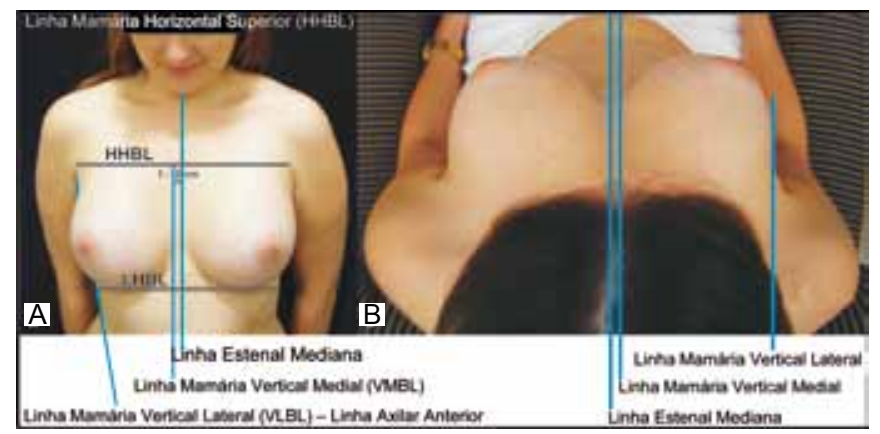

Figura 7 - Visão vertical da mama anatomicamente bonita vista pela paciente, contida entre suas linhas horizontais $H H B L$ e LHBL e verticais $V L B L$ e VMBL, descritas no texto.

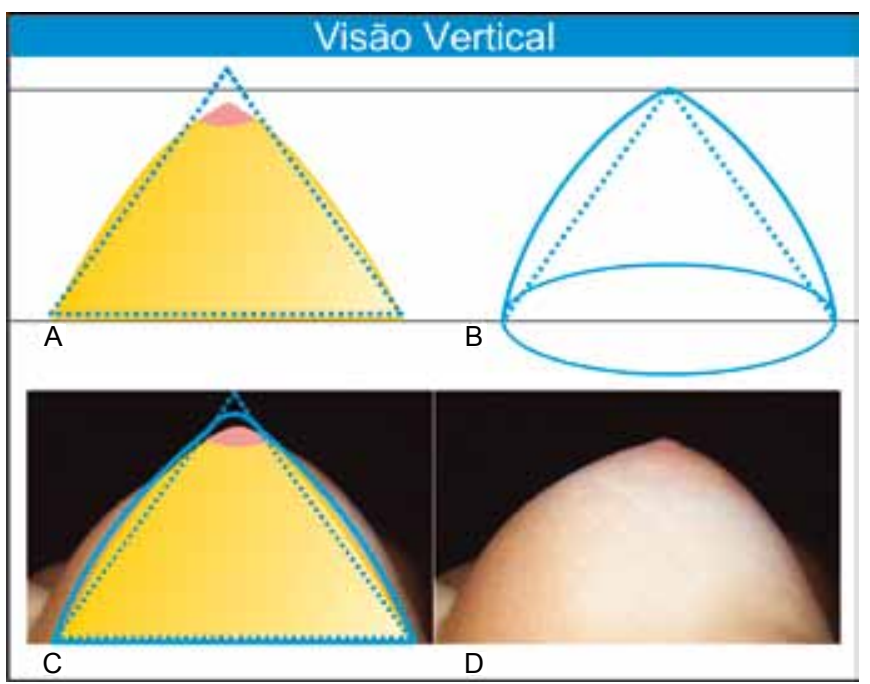

Figura 8 - A: Esquema da visão vertical da mama anatomicamente bonita (amarelo) sobre o cone áureo (vermelho). B: Cone áureo com ligeira curvatura das vertentes, assemelhando-se à mama bonita da Figura 8D. C: Mama anatomicamente bonita na visão vertical subposta aos esquemas. D: Mama bonita com base no cone áureo, sob visão vertical, da própria paciente.

\section{Diagnóstico das formas de mamas}

$\mathrm{Na}$ avaliação clínica completa das alterações estéticas das mamas, incluindo as hipomastias, além da forma em visões frontal, lateral e vertical, é também necessário considerar outros elementos. Na própria mama, deve-se considerar ptose mamária, volume, prolongamentos axilares, prolongamentos peitorais. No tórax, deve-se considerar anatomia torácica com sua inclinação vertical e lateral e curvatura das costelas, presença dos músculos peitorais. E, na aréola papila, deve-se considerar posição, diâmetro, projeção, forma e cor. Finalmente, é necessário observar a simetria.

Neste trabalho, os autores consideraram as formas das mamas e seus volumes contidos nas formas e a base de implantação delas no tórax.
A forma da mama contém e molda seu volume. Vontades individuais, épocas, países ou modismos determinam volumes diversos. Volume e forma são coisas diferentes e devem ser analisados separadamente. Se uma mama contém ou aproxima-se da proporção Phi, ela é bonita, mesmo que o volume não seja.

De acordo com visões frontal, lateral e vertical (base e projeção do cone - sem ptose), é possível observar formas de mamas e suas alterações e classificar as hipomastias e as hipertrofias (Figuras 9 e 10):

- Grupo I (GI): a mama é normal (normomastia). Base, perfil e projeção estão enquadrados de acordo com o "ovoide áureo", o triângulo áureo e o "cone áureo", respectivamente. Ajustada dentro das duas linhas horizontais (HHBL e LHBL) e das duas verticais (VMBL e VLBL) referidas anteriormente. É o grupo controle e descrita em: "Proporções e anatomia da beleza da forma da mama" (Figuras 5 a 8). As características do GI são iguais para ambas as classificações, hipomastias e hipertrofias, estas previamente descritas pelo autor sênior (Figuras 9 e 10$)^{20}$. Esteticamente, não há indicação para o aumento mamário em mamas GI, exceto por anseios pessoais da paciente e ou exigências socioculturais.

- Grupo II (GII), hipomastias: O diâmetro de base da mama é normal ou alargado, mas sem projeção ideal. Não há suficiente volume (Figuras 9 e 10).

- Grupo III (GIII), hipomastias: É o inverso do GII. A projeção do cone é normal ou aumentada e o diâmetro da base é reduzido (Figuras 9 e 10). Não há suficiente volume.

- Grupo IV (GIV), hipomastias: A projeção do cone e sua base são reduzidas ou ausentes. Não há suficiente volume. Amastias incluem-se nesse grupo (Figuras 9 e 10).

Ptose deve ser ignorada para fazer a classificação da

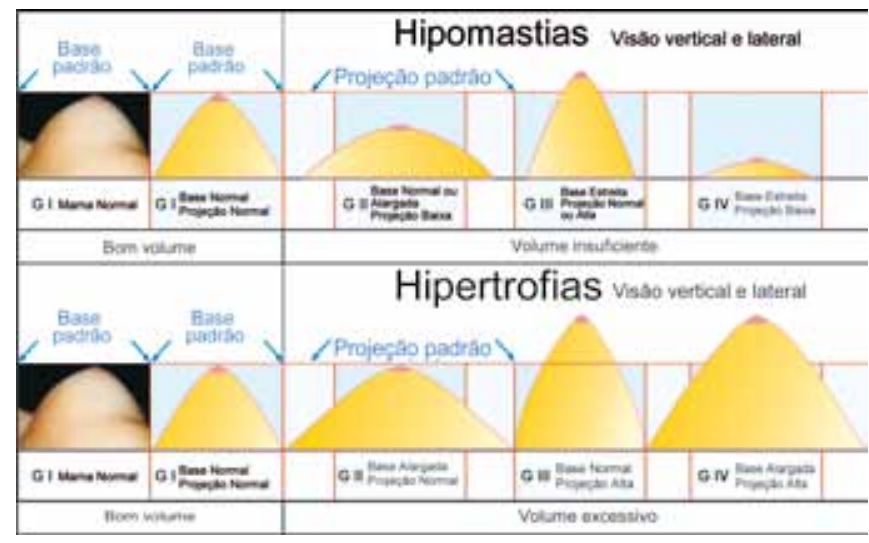

Figura 9 - Esquema da classificação de forma de mamas com hipertrofias e hipomastias e a mama padrão (GI). Esta considerada anatomicamente bonita, sob visão vertical e lateral, sem ptose quanto à forma e ao volume e tocando as linhas horizontais e verticais. 


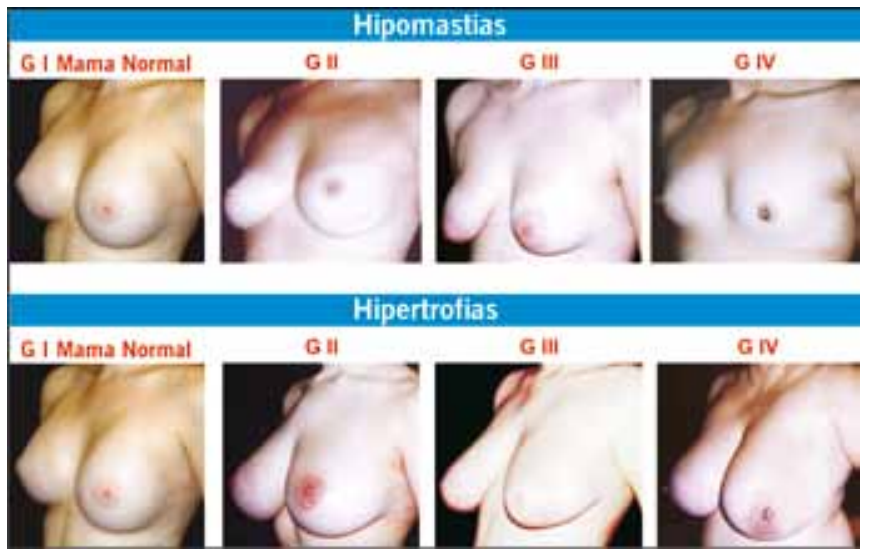

Figura 10 - Fotos de pacientes com as várias formas de hipertrofias e hipomastias descritas no texto e de acordo com os esquemas da Figura 9.

forma. Se presente, a paciente ou o cirurgião, usando a mão espalmada, elevam a mama até a posição ideal, desconsiderando a ptose e procedendo à análise da forma.

\section{Próteses}

Foram desenvolvidas duas formas de próteses Phi, em duas diferentes projeções: as formas PhiA (alta projeção) possuem o padrão anatômico de beleza da mama descrita anteriormente. Em visão frontal, entre os diâmetros horizontal e vertical, há uma proporção de 1 para 1.3, determinada pela proporção Phi entre os diâmetros dos dois círculos parcialmente superpostos (ovoide áureo - Figura 11A). Em visão lateral, do ápice (M) ao bordo inferior (ponto I), e do ápice ao bordo superior, há uma proporção Phi (1/1.618) (triângulo áureo - Figura 11B). Em visão vertical, a relação entre a medida da projeção e da base é Phi (1/1.618) (cone áureo - Figura 11C). Estas próteses são indicadas para casos do GII e GIV hipomastias.

As formas de próteses PhiB (baixa projeção) apresentam projeção diminuída na visão lateral e vertical (Figura 11B) e são indicadas para mamas mais projetadas (GIII hipomastia).

Em visão frontal, cada meio centímetro do segmento vertical (AD) na linha do meridiano da prótese, que variou entre 13 e $15 \mathrm{~cm}$, gerou próteses com 5 diferentes volumes. Se a medida vertical da prótese PhiA de $13 \mathrm{~cm}(280 \mathrm{ml}) \mathrm{de}$ comprimento mudou para $13,5 \mathrm{~cm}$ de comprimento, o volume aumentou $50 \mathrm{ml}$; de $13,5 \mathrm{~cm}$ para $14 \mathrm{~cm}$, gerou mais $55 \mathrm{ml}$; de $14 \mathrm{~cm}$ para $14,5 \mathrm{~cm}$, gerou mais $60 \mathrm{ml}$; de $14,5 \mathrm{~cm}$ para $15 \mathrm{~cm}$, gerou mais $65 \mathrm{ml}$ de volume. Portanto, uma variação de $13 \mathrm{~cm}$ para $15 \mathrm{~cm}$ resulta $230 \mathrm{ml}$ mais volume (PhiA- $15 \mathrm{~cm}$ $=510 \mathrm{ml}$ ). A Indústria Silimed (Brasil) fabricou 10 pares de próteses Phi de acordo com o projeto: 5 pares PhiA e 5 pares

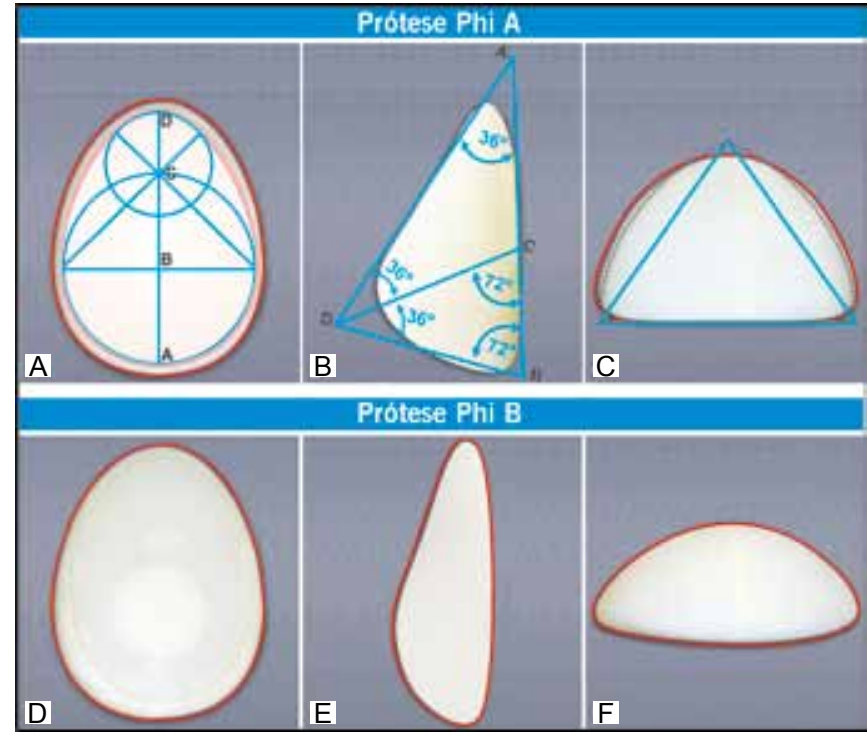

Figuras 11 - A, B e C: Forma de prótese PhiA, nas visões frontal, lateral e vertical, com a proporção Phi em todas elas, utilizáveis nas mamas GII e GIV, hipomastias e reconstrução mamária. As linhas retas foram ligeiramente curvadas e os ápices eliminados. $\boldsymbol{D}, \boldsymbol{E} \boldsymbol{e}$ F: Forma de prótese PhiB, com redução da projeção, nas visões vertical e lateral, utilizáveis em mamas GIII hipomastias.

PhiB, com uma unidade reserva para cada par, a fim de ser utilizada em eventuais necessidades durante as cirurgias.

\section{Casuística}

Dez pacientes do sexo feminino, com idades entre 20 e 45 anos, foram selecionadas entre o quadro de funcionários do Hospital de Base de São José do Rio Preto, SP, Brasil. Estas pacientes eram casos GI que desejavam mais volume, GII, GIII e GIV de hipomastias e uma amastia pós-mastectomia.

As pacientes foram operadas entre outubro de 2008 e maio de 2009 e acompanhadas por 12 meses.

\section{Demarcação pré-operatória e escolha das próteses}

Com a paciente de pé, marcou-se a linha vertical médioesternal, a linha do meridiano mamário (MBL) para cada mama, a linha mamária horizontal inferior (LHBL) e a linha mamária horizontal superior (HHBL) (Figura 12). Marcaram-se também os pontos I direito e esquerdo no cruzamento de MBL e LHBL. A implantação da base da futura mama, sem flacidez, deverá estar entre as duas linhas horizontais em visão frontal e as linhas verticais VLBL e VMBL.

As próteses foram escolhidas baseadas: a) na medida vertical na linha médio -esternal entre as linhas LHBL e HHBL (na paciente da Figura 12 é de 13,5 cm), b) na classificação das hipomastias proposta nesse estudo. 


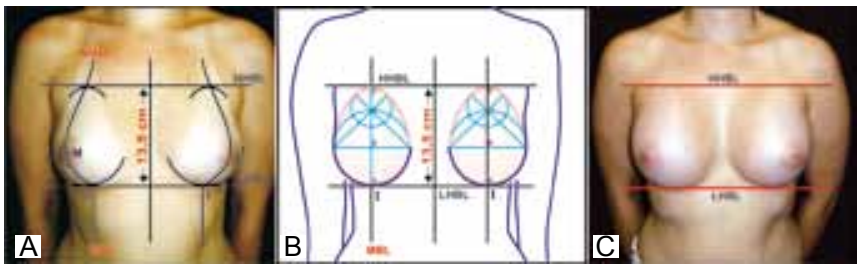

Figura 12-A: Na visão frontal, a escolha da prótese pela medida vertical da paciente entre as linhas HHBL e LHBL sobre a linha médio-esternal. Nesta paciente, $13.5 \mathrm{~cm}$. B: Esquema da mesma paciente e da base da prótese escolhida de $13.5 \mathrm{~cm}$ no eixo vertical, sob visão frontal e dentro das linhas e pontos descritos no texto.

C: A mesma paciente após a inclusão das próteses.

A medida vertical das pacientes pré-selecionadas variou entre 12,5 e 15,5 cm (Figura 12B), mas foram escolhidas de acordo com a medida vertical das próteses industrializadas disponíveis para este estudo (entre 13 e $15 \mathrm{~cm}$ ).

Para pacientes dos grupos GIV e GII hipomastias com baixa projeção, as próteses PhiA (alta projeção) foram utilizadas. Para pacientes GIII hipomastias com alta projeção, as próteses PhiB (baixa projeção) foram empregadas. A visão lateral foi suficiente para observar as projeções mamárias.

Quando havia ptose, a manobra de levantamento da mama, descrita previamente, foi realizada para o diagnóstico da forma e projeção mamária.

\section{Cirurgia}

Todas as pacientes foram operadas sob anestesia geral. No início da cirurgia, injetou-se $1 \mathrm{~g}$ dehidrocortisona e.v.endovenosa e, no final do ato cirúrgico, $10 \mathrm{mg}$ de dexametasona.

As incisões e excisões de pele foram escolhidas de acordo com a necessidade de remover ou não excessos de pele para correção de ptose. Incisões axilares não foram utilizadas. O descolamento foi realizado na posição retroglandular, na metade inferior da loja e sob a fáscia muscular ${ }^{21} \mathrm{em}$ sua metade superior, exceto na primeira paciente (GII-hipomastia). Nesta paciente, o descolamento total foi realizado em posição retroglandular. A amplitude da base da loja foi aproximadamente $0,5 \mathrm{~cm}$ mais extensa que a da base da prótese (Figura 13). $\mathrm{O}$ meridiano mamário (MBL) e o meridiano da prótese foram coincidentes.

Cada prótese foi lavada com solução salina e colocada imediatamente dentro de luva plástica. Foi implantada, e assim que removida a luva, foi ajustada exatamente em sua posição adequada dentro da loja. Quando houve dificuldades para seu posicionamento, uma valva de fibra óptica e uma espátula foram utilizadas para checar a posição (Figura 14). Antes do final da cirurgia, foi introduzido um cateter em cada loja cirúrgica. Injetou-se $30 \mathrm{ml}$ por loja de uma solução de
$60 \mathrm{ml}$ contendo soro fisiológico, $10 \mathrm{mg}$ de dexametasona, $20 \mathrm{ml}$ de lidocaína, $1 \mathrm{ml}$ de adrenalina $1 / 1000$ e 5000 UI de hialuronidase. Retirados os cateteres e com o objetivo de espalhar melhor a solução realizou-se uma suave massagem sobre as mamas.



Figura 13 - Loja pronta para receber a prótese, sob a fáscia do músculo grande peitoral, na metade superior, marcada com a linha branca. O perímetro da loja $0,5 \mathrm{~cm}$ mais amplo que o perímetro da base da prótese.

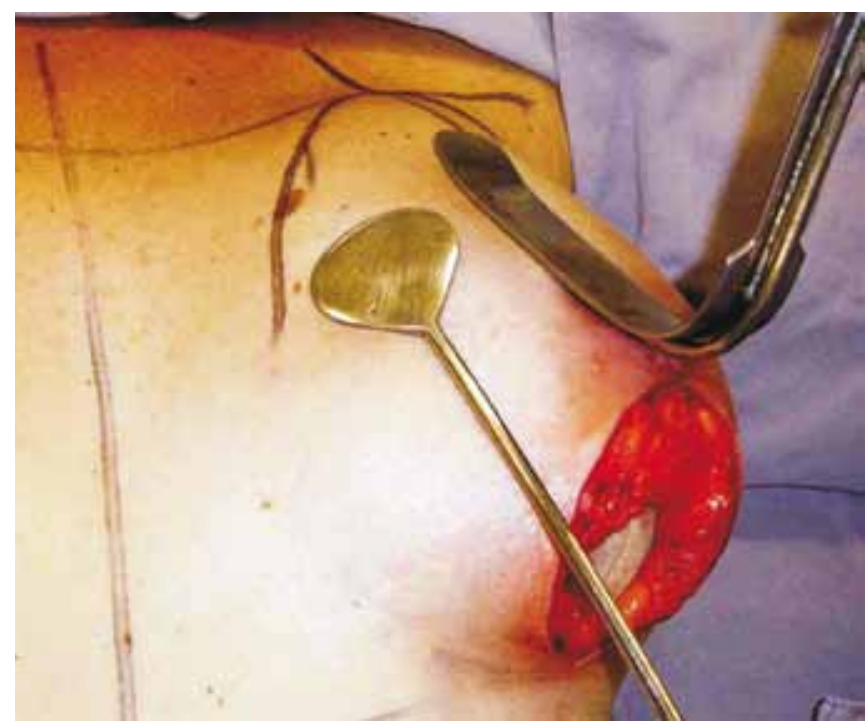

Figura 14 - Valva de fibra óptica e espátula para checar a distribuição da prótese na loja. Excisão vertical de fuso de pele. Paciente da Figura 18. 


\section{RESULTADOS}

As pacientes não apresentaram nenhuma complicação pósoperatória importante. A primeira paciente operada, do GII hipomastia, era magra e sua prótese foi toda colocada em nível retroglandular. Apresentou a prótese esquerda mais evidente em seu bordo superior e recebeu, então, suave compressão no pólo superior das mamas com faixa torácica durante 2 meses. Após este período, o resultado normalizou-se.

Graus de satisfação das pacientes foram avaliados ao serem indagadas e emitiram notas de 0 a 10 para o resultado final. A média aritmética de suas notas foi 9,9. Também outros cirurgiões plásticos avaliaram cada resultado final, emitindo notas de 0 a 10 e a média aritmética foi de 9,0. No estudo, os autores consideraram que as próteses Phi apresentaram melhores resultados finais de forma que os obtidos com as próteses convencionais.

As Figuras 15 a 21 são de pacientes que receberam as formas de próteses Phi descritas. A Figura 15 (GI) é de paciente que desejou aumento, mesmo tendo mamas de forma e volume adequados para o país que vive (Brasil). A Figura 16 referese a paciente do grupo GII-hipomastia. No caso ilustrado na Figura 17, houve troca de próteses convencionais por PhiA e melhora das cicatrizes. A Figura 18 refere-se a paciente do grupo GIII-hipomastia. A Figuras 19 ilustra caso de paciente classificada como GIV-hipomastia, depois de pexia mamária há 5 anos e insatisfação com o volume e forma mamária. As Figuras 20 e 21 são de paciente submetida à reconstrução mamária bilateral com inclusão prévia de expansor à direita durante 90 dias e troca por prótese, mais adenomastectomia profilática à esquerda e inclusão de prótese.

Foram utilizadas todos os 5 pares de próteses PhiA e os 5 pares PhiB. Não houve necessidade de usar nenhuma prótese suplementar.

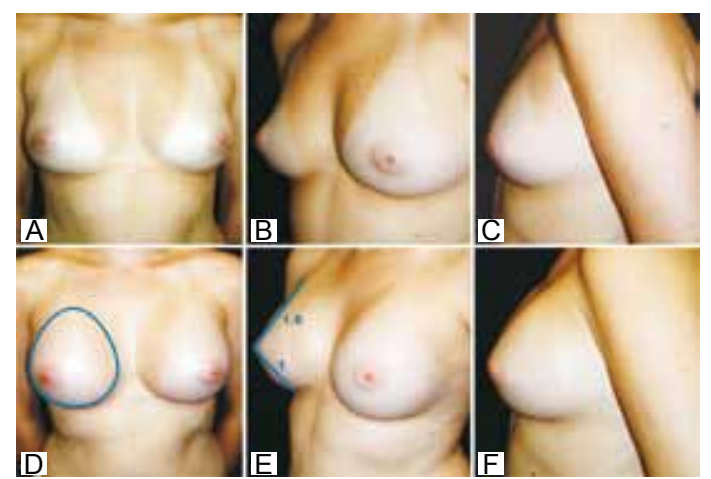

Figura 15-Mesma paciente da Figura 12. Esta paciente pode ser classificada como GI, entretanto desejou maior volume.

Pré-operatório e pós-operatório de 6 meses, com a proporção Phi estabelecida em todas as visões. Próteses PhiA 13,5 cm no eixo vertical $(330 \mathrm{ml})$. Incisão de pele submamária.

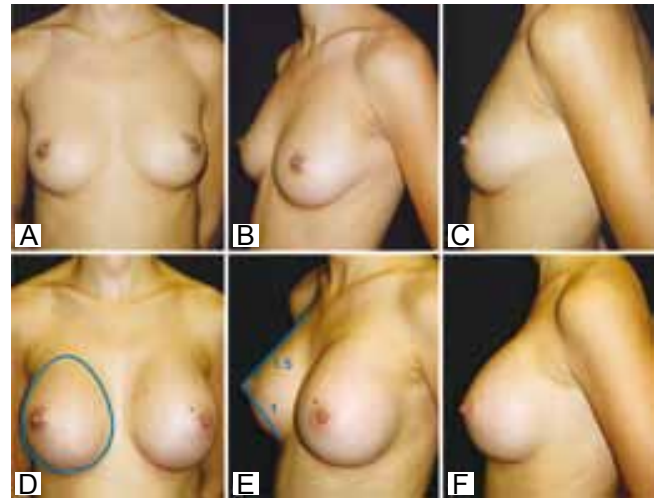

Figura 16 - Pré-operatório de paciente do grupo GII de forma de hipomastia e pós-operatório de 12 meses. Prótese PhiA $13 \mathrm{~cm}$ (280 ml) na medida vertical da base, igual à medida vertical da paciente entre as linhas HHBL e LHBL. Incisão submamária. Medidas próximas da proporção Phi.

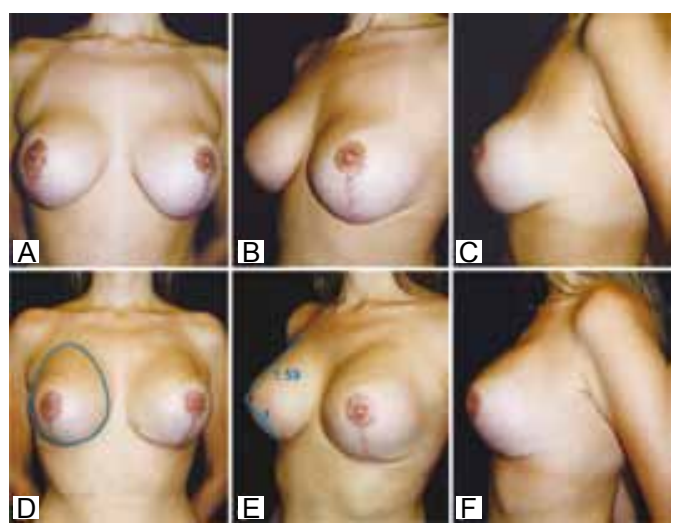

Figura 17 - Pré-operatório e 5 meses de pós-operatório com ressecção de cicatrizes em Tinvertido e troca de próteses por PhiA $14.5 \mathrm{~cm}$ no eixo vertical $(445 \mathrm{ml})$. Observe a aproximação da proporção Phi nas visões lateral e frontal.

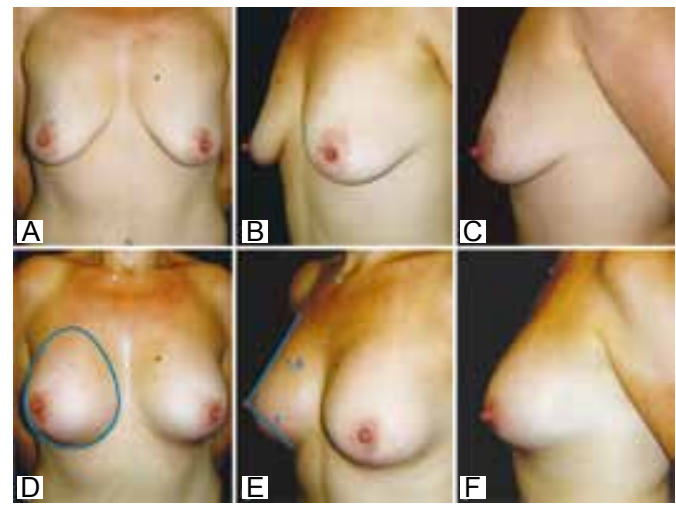

Figura 18 - Pré-operatório de paciente do GIII de forma de hipomastia com moderada ptose e 6 meses de pós-operatório com ressecção fusiforme vertical de pele do ponto I até a papila. Prótese utilizada PhiB $15 \mathrm{~cm}(435 \mathrm{ml})$ na medida vertical, igual à medida vertical da paciente. Proporção Phi presente. 


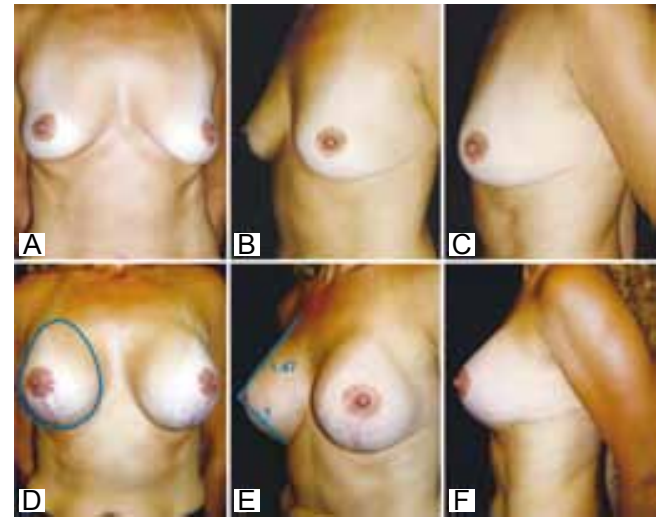

Figura 19 - Paciente do GIV de forma de hipomastia e 10 meses de pós-operatório, que foi submetida à mastopexia com cicatrizes em L há 5 anos. Utilizadas próteses PhiA $14 \mathrm{~cm}(385 \mathrm{ml})$, igual à medida vertical do tórax entre $L H B L$ e HHBL. Proporções mais distantes de Phi, notando-se uma visão lateral menos bonita.



Figura 20 - Paciente do GIV de forma de hipomastia (lado direito) pós-mastectomia com cicatriz transversal. Reconstrução após expansão e troca do expansor por prótese PhiA $14,5 \mathrm{~cm}$ $(445 \mathrm{ml})$. Medida vertical igual à medida vertical da paciente na linha médio-esternal entre as linhas HHBL e LHBL. Reconstrução da papila e tatuagem de aréola. A mesma incisão transversal foi usada na mama esquerda para adenomastectomia profilática e utilização de prótese PhiA 14,5 cm (445 ml). Relocação da aréola-papila por retalho.



Figura 21 - Pós-operatório da paciente da Figura 20. Proporções próximas de Phi.

\section{DISCUSSÃO}

Sob visão frontal, a variação da medida vertical das mamas de forma bonita leva a variações proporcionais nas outras medidas, gerando volumes diferentes se mantida a proporção Phi entre elas. Por isso, com base apenas na medida vertical entre a HHBL e LHBL, sobre a linha médio-esternal da paciente, e na projeção do seu cone mamário (GII-III-IV), é possível escolher as próteses PhiA ou PhiB.

Cada paciente tem sua medida vertical, que determina a extensão vertical da prótese a ser escolhida, e a classificação das hipomastias determina o uso de prótese PhiA ou PhiB.

As linhas horizontais HHBL e LHBL, e as verticais VMBL e VLBL são também indicativas para escolha da extensão da base de expansores de pele em reconstrução mamária:

- Mamas GI - cujas proporções de forma e volume são equilibradas, supostamente não necessitam de correção, mas se houver o desejo por mais volume, utiliza-se próteses PhiA para muita projeção ou PhiB para projeção menor. A marcação utilizada é a mesma;

- Mamas GII hipomastias - as pacientes não apresentam projeção e a forma de prótese PhiA (alta projeção) é a melhor indicação;

- Mamas GIII hipomastias - as pacientes apresentam projeção mamária e não é necessário aumentá-la mais. É preconizada a forma de prótese de menor projeção (PhiB). Nas pacientes do GIII hipomastias, a projeção mamária pode ser diminuída realizandose uma incisão longitudinal no meridiano da glândula mamária, causando o "desabamento" de seus segmentos lateral e medial no diâmetro horizontal. Esta manobra pode alterar a escolha do perfil da prótese;

- Mamas GIV hipomastias e amastias pós-mastectomias - as pacientes têm pouca ou nenhuma mama, então a forma de prótese indicada é de alta projeção (PhiA). Somente ela dará forma final bonita e volume adequado, como foi demonstrado na paciente das Figuras 20 e 21.

As próteses Phi são preenchidas com silicone ligeiramente mais duro que as convencionais e cobertas com poliuretano. Apesar disso, a sensação na palpação de mama natural não foi prejudicada. A experiência do autor sênior ${ }^{22}$ com as próteses cobertas com poliuretano revelou que estas não se deslocam no interior de suas lojas e fixam-se aos tecidos cruentos como velcro, e a incidência de contratura capsular é menor.

Os autores sugerem que a cobertura de poliuretano é necessária para evitar deslocamentos da prótese dentro de sua loja, prevenindo deformidades. Como a prótese Phi tem forma irregular, ela não deve se mover dentro da loja. Trabalhos futuros devem ser realizados a fim de determinar a veracidade da observação.

A porção superior da prótese foi colocada sob a fáscia do músculo peitoral maior, porque assim ela acompanha melhor 
a inclinação do tronco superior devido à compressão ânteroposterior exercida pela maior rigidez da fáscia.

Posicionar e ajustar a prótese sem dobras dentro da loja cirúrgica é importante, a prótese coberta com poliuretano fixando-se aos tecidos cruentos não se desloca no período pósoperatório. Qualquer dobra não poderá ser desfeita mais tarde.

A colocação da prótese não deve ser realizada com força exagerada através de pequenas incisões, deformidades permanentes podem ocorrer em sua forma.

A solução injetada através de cateter ao final da cirurgia contendo dexametasona, lidocaína, adrenalina e hialuronidase reduz dor e edema no pós-operatório imediato, além de reações inflamatórias, fibrose excessiva e consequente incidência de contratura capsular.

Durante 2 meses, a prótese de poliuretano não deve receber massagens ou traumas de qualquer tipo. Ela pode se desgarrar dos tecidos cruentos e provocar hematomas, seromas, fibrose e contratura capsular.

Eventualmente, se a paciente deseja mamas maiores que as cabíveis entre as linhas horizontais e verticais descritas, o cirurgião pode aumentar a medida vertical da prótese. Para isto deve-se aumentar o descolamento abaixo do sulco inframamário (LHBL). Por outro lado, se a paciente deseja mamas menores, o descolamento deve ser ajustado acima do sulco inframamário. $\mathrm{O}$ descolamento superior não muda em ambos os casos.

Em visão frontal, se há ptose, as medidas do diâmetro vertical e horizontal da base da mama invertem-se entre si e o pólo superior apresenta-se vazio. Além da ressecção dos excedentes de pele, as formas de prótese PhiA e PhiB são as melhores opções para preencher o vazio do colo e inverter novamente os diâmetros para a proporção ideal de 1/1.3.

Os autores consideram que muitas pacientes se satisfazem apenas com o aumento mamário e fica prejudicada a percepção de uma mama com formas ideais e proporcionais. Entretanto, quando as pacientes do estudo compararam a forma final de suas mamas com resultados finais de forma de pacientes que receberam próteses convencionais, especialmente aquelas que tiveram suas próteses trocadas pelas PhiA e PhiB (duas pacientes), demonstraram maior grau de satisfação.

Volumes de próteses Phi não devem ser comparados aos de próteses convencionais para a escolha. A base da prótese Phi é mais extensa e o volume correspondente às convencionais será sempre maior.

Os ângulos do triângulo áureo naturalmente foram abolidos para a confecção das próteses.

\section{CONCLUSÃO}

Observando a forma da mama bonita, sob perspectiva tridimensional (visões frontal, lateral e vertical), e utilizando a proporção Phi, os autores concluíram que a forma ideal de uma prótese mamária deve conter esta proporção. Esta forma de prótese é capaz de corrigir todos os casos de hipomastia mamária, incluindo reconstruções, e permite bons resultados estéticos. A escolha delas baseia-se na medida vertical da paciente e na classificação das hipomastias.

\section{REFERÊNCIAS}

1. Vázquez G. Modificación del implante anatómico de gel de silicona con cubierta de poliuretano. Cir Plást Iberlatinamer. 2005;31(3):193-8.

2. Capozzi A, Pennisi VR. Clinical experience with polyurethane-covered gel-filled mammary prostheses. Plast Reconstr Surg. 1981;68(4):512-20.

3. Eyssen JE, von Werssowetz AJ, Middleton GD. Reconstruction of the breast using polyurethane-coated prostheses. Plast Reconstr Surg. 1984;73(3):415-21.

4. Mira JA. Anatomic asymmetric prostheses: shaping the breast. Aesthet Plast Surg. 2003;27(2):94-9.

5. Fruhstorfer BH, Hodgson EL, Malata CM. Early experience with an anatomical soft cohesive silicone gel prosthesis in cosmetic and reconstructive breast implant surgery. Ann Plast Surg. 2004;53(6):536-42.

6. Peck H, Peck S. A concept of facial esthetics. Angle Orthod. 1970;40(4):284-318.

7. Marquardt SR. Marquardt beauty analysis (web site). Available at: http:// www.beautyanalysis.com. Accessed March 15th, 2010.

8. Etcoff NL. Psychology. Beauty and the beholder. Nature. 1994;368(17):186-7

9. Etcoff NL. A lei do mais belo. Rio de Janeiro:Editora Objetiva;1999.

10. Ricketts RM. The biologic significance of the divine proportion and Fibonacci series. Am J Orthod. 1982;81(5):351-70.

11. Livio M. La proporción áurea, la historia de PHI, el numero más sorprendente del mondo. Barcelona:Editorial Ariel;2005.

12. Brown D. The Da Vinci code. Special Illustrated Edition. GMT Editores; 2004.

13. Eco U. Storia della belleza. Milão:Bompiani;2004.

14. Atalay B. Math and the Mona Lisa, the art and science of Leonardo da Vinci. New York:Harper Collins;2004.

15. Ono E, Walter-Porto COT, Medici-Filho E, Moraes LC, Moraes MEL, Castilho JCM. Análise da proporção áurea em indivíduos dolico, braqui e mesofaciais, por meio de radiografias cefalométricas laterais. Rev Odonto Ciênc. 2007;22(56):154-9.

16. Gil CTLA, Médici Filho E. Estudo da proporção áurea na arquitetura craniofacial de indivíduos adultos com oclusão normal, a partir de telerradiografias axiais, frontais e laterais. Ortodontia. 2002;35(2):69-85.

17. Preston JD. The golden proportion revisited.JEsthetDent. 1993;5(6):24751.

18. Bashour M. History and current concepts in the analysis of facial attractiveness. Plast Recontr Surg. 2006;118(3):741-56.

19. Langlois JH, Roggman LA, Casey RJ, Ritter JM, Rieser-Danner LA, Jenkins VY. Infant preferences for attractive faces: rudiments of a stereotype? Developmental Psychology 1987;23(3):363-9.

20. Bozola AR. Breast reduction with short L scar. Plast Reconstr Surg. 1990;85(5):728-38.

21. Graf RM, Bernardes A, Rippel R, Araujo LR, Damasio RC, Auersvald A. Subfascial breast implant: a new procedure. Plast Reconstr Surg. 2003;111(2):904-8.

22. Bozola AR, Bozola AC, Carrazzoni RM. Inclusão de próteses mamárias de silicone-poliuretano. Rev Soc Bras Cir Plast. 2006;21(1):18-22.

Correspondência para: 